\title{
EVALUATION OF OUTCOME OF COCHLEAR IMPLANTED CHILDREN AFTER TWO YEARS OF REHABILITATION IN ALEXANDRIA
}

Fathy Ahmed Abdel Baky, Riham Mohamed El Maghraby, Engy Samy Abdel Hafiz El Hakeem, Heba Mohamed Gamal Eldin Elroumy Department of Otorhinolaryngology, Phoniatrics Unit, Faculty of Medicine, Alexandria University

\section{Introduction}

Hearing loss is the most common sensory deficit in children. Patients who suffer from severe to profound sensori-neural hearing loss face challenges in developing spoken language because of inability to detect acoustic-phonetic cues that are crucial for speech recognition. Thus, it is important to minimize the duration of auditory deprivation between the beginning of bilateral hearing loss and intervention using hearing devices such as hearing aids and cochlear implants. In order to prevent serious impacts of hearing loss on children, they should also receive appropriate auditory rehabilitation including counseling and comprehensive auditory training program.

\section{Aim of the work}

- To Assess the progress and overall improvement of all aspects of language acquisition. - To demonstrate the relationship of the various factors affecting the degree of improvement and possible outcome.

\section{Subjects and Methods}

The study was conducted on 33 children with cochlear implantation undergoing auditory training at the Unit of Phoniatrics, in the outpatient clinic of Alexandria Main University hospital; within the period of six months starting 1/7/2019.

The subjects of the study were assessed before and after implantation by a set protocol of assessment including:

Elementary diagnostic procedures

-History taking

-Audiological evaluation (Pure tone audiometry)

-Lip reading and sound detection, Auditory perceptual assessment (APA)

-language rehabilitation prior to implantation: (Duration, and Regularity)

Clinical diagnostic procedures:

-Formal language testing using: Arabic language test

-Psychometric evaluation

Post Cochlear implantation children were reassessed using same protocol as pre-Cochlear implantation in addition to:

-Operative history.

- Speech and language rehabilitation after implantation: (Place, Duration) and type of parents' involvement; active / passive; and their commitment to required tasks).

\section{Results}

Table 1: Distribution of all studied cases according to demographic data

\begin{tabular}{|l|l|l|}
\hline \multirow{2}{*}{ Age of hearing loss (months) } & Min. - Max. & $0.0-48.0$ (months) \\
\cline { 2 - 3 } & Median (IQR) & $15.0(8.0$ to 20.0) \\
\hline \multirow{3}{*}{ Causes of Hearing loss (n=33) } & Meningitis & $2(6.06 \%$ \\
\cline { 2 - 3 } & Otitis Media & $1(3.03 \%)$ \\
\cline { 2 - 3 } Age of hearing aids fitting (months) & Unknown & $30(90.9 \%)$ \\
\cline { 2 - 3 } & Min - Max. & $1.0-21.0($ months) \\
\hline \multirow{4}{*}{ Laterality of hearing aids (n=33) } & Binaural & $9.0(5.0$ to 13.50$)$ \\
\cline { 2 - 3 } & Mono aural left HA & $2(60.6 \%)$ \\
\cline { 2 - 3 } & Mono aural right & $10(30 \%)$ \\
\cline { 2 - 3 } & No hearing aids & $2(6.1 \%)$ \\
\hline \multirow{3}{*}{ Regularity of using hearing aids (n=33) } & Iregular & $6(18.2 \%)$ \\
\cline { 2 - 3 } & No hearing aids & $2(6.1 \%)$ \\
\cline { 2 - 3 } & Regular & $25(75.7 \%)$ \\
\hline \multirow{2}{*}{ Parents' involvement (n=33) } & Negative & $8(24.2 \%)$ \\
\cline { 2 - 3 } & Positive & $25(75.8 \%)$ \\
\hline
\end{tabular}

Table 2: Distribution of all studied cases according to surgical history

\begin{tabular}{|l|l|l|}
\hline $\begin{array}{l}\text { Age of patients at Cochlear } \\
\text { implantation CI (year) }\end{array}$ & Min. - Max. & $0.58-7.0$ years \\
\cline { 2 - 3 } Laterality of Implant (n=33) & Median (IQR) & $4.0(2.5$ to 5.0$)$ \\
\hline \multirow{3}{*}{ Implant brand (n=33) } & Unilateral & $33.0(100 \%)$ \\
\hline \multirow{3}{*}{ Site of Insertion (n=33) } & Cochlear & $7(21.21 \%)$ \\
\cline { 2 - 3 } & MED-EL & $22(66.67 \%)$ \\
\cline { 2 - 3 } & Oticon & $4(12.12 \%)$ \\
\hline & Boney cochleostomy & $14(42.4 \%)$ \\
\cline { 2 - 3 } & Extended round window & $9(27.3 \%)$ \\
\cline { 2 - 3 } & Round window & $10(30.3 \%)$ \\
\hline
\end{tabular}

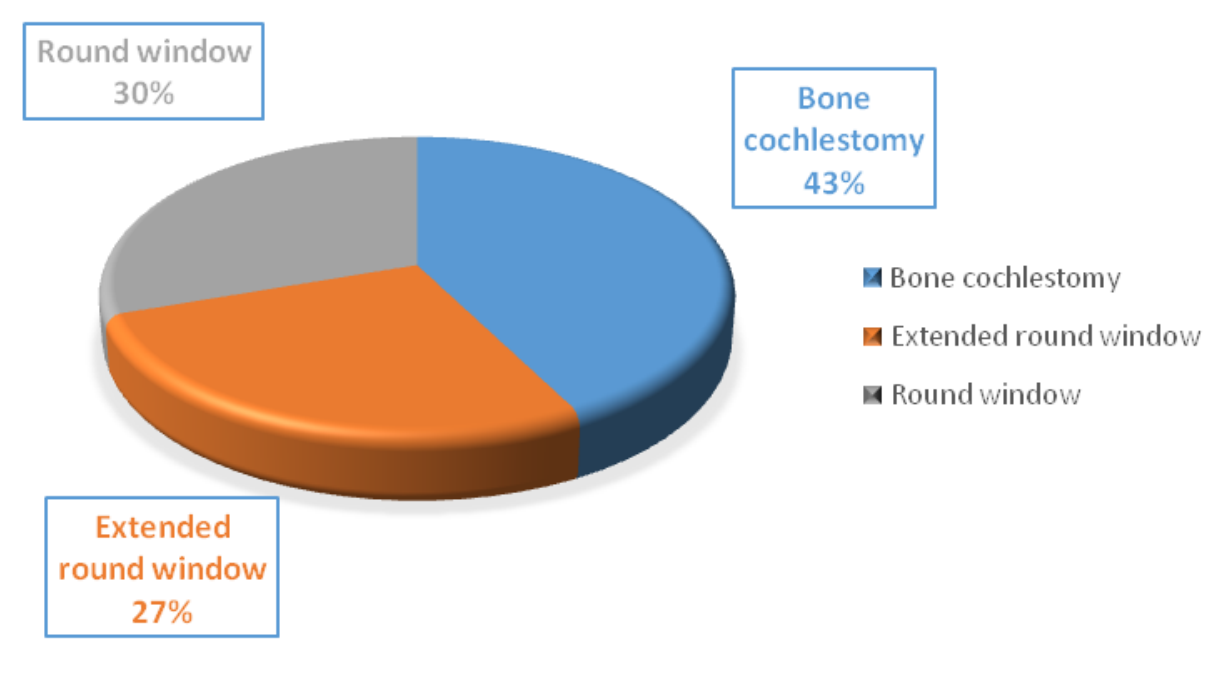

Figure: Distribution of all cases according to site of implantation

\section{Conclusion}

The progress of newborn hearing screening gives advantages in terms of therapeutic provision of children with hearing prostheses.

This study emphasizes the importance of a detailed knowledge of the history of each case to predict and influence the success of rehabilitation following $\mathrm{CI}$.

2021 OAlexandria Faculty of Medicine
CC-BY-NC

